\title{
A case of Raine syndrome presenting with facial dysmorphy and review of literature
}

\author{
Jayesh Sheth ${ }^{1 *}$, Riddhi Bhavsar ${ }^{1}$, Ajit Gandhi ${ }^{2}$, Frenny Sheth ${ }^{1}$ and Dhairya Pancholi ${ }^{1}$
}

\begin{abstract}
Background: Raine syndrome (RS) - an extremely rare autosomal recessive genetic disorder, is caused by a biallelic mutation in the FAM20C gene. Some of the most common clinical features include generalized osteosclerosis with a periosteal bone formation, dysmorphic face, and thoracic hypoplasia. Many cases have also been reported with oro-dental abnormalities, and developmental delay. Most of the cases result in neonatal death. However, a few non-lethal RS cases have been reported where patients survive till adulthood and exhibits a heterogeneous clinical phenotype. Clinical diagnosis of RS has been done through facial appearance and radiological findings, while confirmatory diagnosis has been conducted through a molecular study of the FAM20C gene.

Case presentation: A 6-year-old girl was born to healthy third degree consanguineous parents. She presented with facial dysmorphy, delayed speech, and delayed cognition. Radiography showed small sclerotic areas in the lower part of the right femur, and an abnormally-shaped skull with minimal sclerosis in the lower occipital region. Computer tomography scan of the brain revealed mild cortical atrophy, and MRI scan of the brain showed corpus callosal dysgenesis with the absence of the rostral area. Chromosome banding at 500 band resolution showed a normal female karyotype. No quantitative genomic imbalance was detected by aCGH. Further study conducted using Clinical Exome Sequencing identified a homozygous missense variation c.1228 T>A (p.Ser410Thr) in the exon 6 of FAM2OC gene - a likely pathogenic variant that confirmed the clinical diagnosis of RS. The variant was confirmed in the proband and her parents using Sanger sequencing. Prenatal diagnosis during subsequent pregnancy revealed heterozygous status of the fetus, and a normal carrier child was delivered at term.

Conclusions: The syndrome revealed markedly variable presentations such as facial dysmorphy and developmental delay, and was localized to diffuse bone osteosclerosis. Clinical indications, striking radiological findings and molecular testing of FAM2OC gene confirmed the diagnosis of RS. A rarity of the disorder and inconsistent phenotype hindered the establishment of genotype-phenotype correlations in RS. Therefore, reporting more cases and conducting further research would be crucial in defining the variable radiologic and molecular defects of the lethal and non-lethal forms of this syndrome.
\end{abstract}

Keywords: Case report, Developmental delay, Facial dysmorphy, FAM20C gene, Osteosclerosis, Raine syndrome

\section{Background}

Raine syndrome (OMIM \#259775) is also known as osteosclerotic bone dysplasia. With the estimated prevalence of $<1$ in 1,000,000, the disease is categorized as a rare autosomal recessive disorder. A mutation in the FAM20C (Family with sequence similarity 20, member $\mathrm{C}$ ) gene (OMIM* 611061) located on chromosome 7 p22.3 is responsible for the disease [1]. This gene

\footnotetext{
* Correspondence: jshethad1@gmail.com

${ }^{1}$ FRIGE's Institute of Human Genetics, FRIGE House, Jodhpur Gam Road,

Satellite, Ahmedabad 380015, India

Full list of author information is available at the end of the article
}

encodes a member, which is a Golgi casein kinase and has an $\mathrm{S}-\mathrm{x}-\mathrm{E} / \mathrm{pS}$ consensus motif (where $\mathrm{S}$ is serine, $\mathrm{x}$ is any amino acid and E/pS can be Glutamic acid or phosphoserine) that phosphorylates the serine residues of the extracellular proteins called several secretory calcium-binding-phosphoproteins (SCPP). The biomineralization of bones and teeth is carried out by a small integrin-binding ligand - N-linked glycoproteins (SIBLINGs), an SCPP due to its high affinity for calcium [2]. Also, FAM20C phosphorylates the C-terminal dentin matrix protein 1 (Dmp1) and makes it a highly negatively charged domain, which in turn recruits 
calcium ions and help the mineral deposition [3]. A mutation in the FAM20C gene is reported to cause down-regulation in Dmp1 mRNA expression and upregulation of the fibroblast growth factor 23 (FGF23) mRNA expression, which results in FGF23-related hypophosphatemia in RS [4]. FGF23 maintains phosphate homeostasis in the body and stops reabsorption of excess phosphate in the bloodstream.

In 1989, a unique case of a neonate born with a set of clinical indications including cleft palate, hypoplastic nose, low set ears, gum hyperplasia, microcephaly, exophthalmos, and diffuse osteosclerosis of the bones was described by Raine et al. The proband expired within $3 \mathrm{~h}$ of her birth. This presented the first case of RS [5]. The term 'Raine syndrome' was proposed when 2 other cases of a rare neonatal lethal sclerotic bone disorder exhibiting similar clinical presentations were reported [6]. RS is characterized by generalized osteosclerotic bone dysplasia, periosteal bone formation, and a distinct facial phenotype. To the best of our knowledge, approximately 24 cases of RS with homozygous or compound heterozygous missense mutation, microdeletion, whole gene deletion, and splice-site mutations in the FAM20C gene have been reported till the year 2017 [5-28].

Over time, it has been observed that RS has a broader phenotypic spectrum. Many cases of RS initially described were shown to be lethal within the first few weeks of life [5, 14, 21, 24]. However, cases have been reported where the patient lived up to teenage years or adulthood, which suggests the presence of non-lethal form of RS [8, 11, 12, 29, 30]. Heterogeneity of the clinical phenotypes ranging from mild to severe manifestation has also been observed in RS. Certain lethal cases have been described with severe osteosclerosis, brachycephaly and choanal atresia in the affected patients $[9,24]$. On the other hand, despite having such anomalies, the patient survived till childhood [13]. Other clinical features like microcephaly, delayed language and fine motor skills, periapical abscesses, gingival hyperplasia and open bite malocclusion have also been observed to be associated with RS [8].

The present case reports a child exhibiting non-lethal form of RS portraying craniofacial abnormalities, osteosclerosis, and developmental delay. Upon investigation, a homozygous missense variant in the FAM20C gene was observed. During subsequent pregnancy, the parents and the fetus were heterozygous for the said variant.

\section{Case presentation}

\section{Clinical features}

A 6-year-old girl, was born to healthy parents with third-degree consanguinity. This family of Indian origin was referred for investigation and genetic counseling for facial dysmorphy with global developmental delay, and behavioral issues of the proband. Maternal history revealed intra-uterine growth retardation during the antenatal period that ended with a full-term female baby born through cesarean section due to breach presentation. Her birth weight was $2.75 \mathrm{~kg}$.

The proband had normal feeding history, and there were no neonatal concerns. At approximately 5 months of age, the proband attained her neck control. She could stand with support by 3 years and could walk independently by 5 years of age. She could utter only a few bi-syllable words. Despite being hyperactive, no abnormal hand movements were observed. She had a history of two episodes of febrile seizures at 8 months and 2 years of age. During these episodes, she was observed with other symptoms such as cough, cold, and fever. Her physical examination revealed craniofacial disproportion, which included microcephaly with a narrow bifrontal diameter and a flat forehead. Other anomalies such as facial dysmorphy including epicanthal folds, hypertelorism, depressed and low nasal bridge with bulbous nasal tip, flaring nares, prominent philtrum, and pointed chin were also observed (Fig. 1a). However, no oro-dental anomalies were observed in the proband.

She also had hallux valgus with increased gap between the first and second toes (sandal gap deformity), clinodactyly of toes, and pes planus (flat feet). No other deformity of the trunk or limbs was observed. She had dark pigmentation in armpits, genitalia, and on the knuckles. She had mild hypotonia with brisk and deep tendon reflexes. Her expressive communication was good but her verbal communication was affected. Her social responses seemed to be fair. The proband was diagnosed with attention deficit hyperactivity disorder (ADHD) with psychomotor developmental delay. Sample collection and written informed consent was obtained according to the need of the institutional ethics committee in accordance with Helsinki declaration. CARE guidelines were followed for reporting.

\section{Radiological and haematological investigations}

On investigation, low hemoglobin with normal serum calcium $(10 \mathrm{mg} / \mathrm{dl})$ and Vitamin B12 levels were detected. Her sleep electroencephalogram (EEG) was normal. Radiographic studies of the proband showed small sclerotic areas in the lower part of the right femur, and abnormally shaped skull with minimal sclerosis in the lower occipital region (Fig. 1b). However, the bone density was normal and there were no features of fracture or dislocation of the bones. Corticomedullary differentiation was maintained and no 


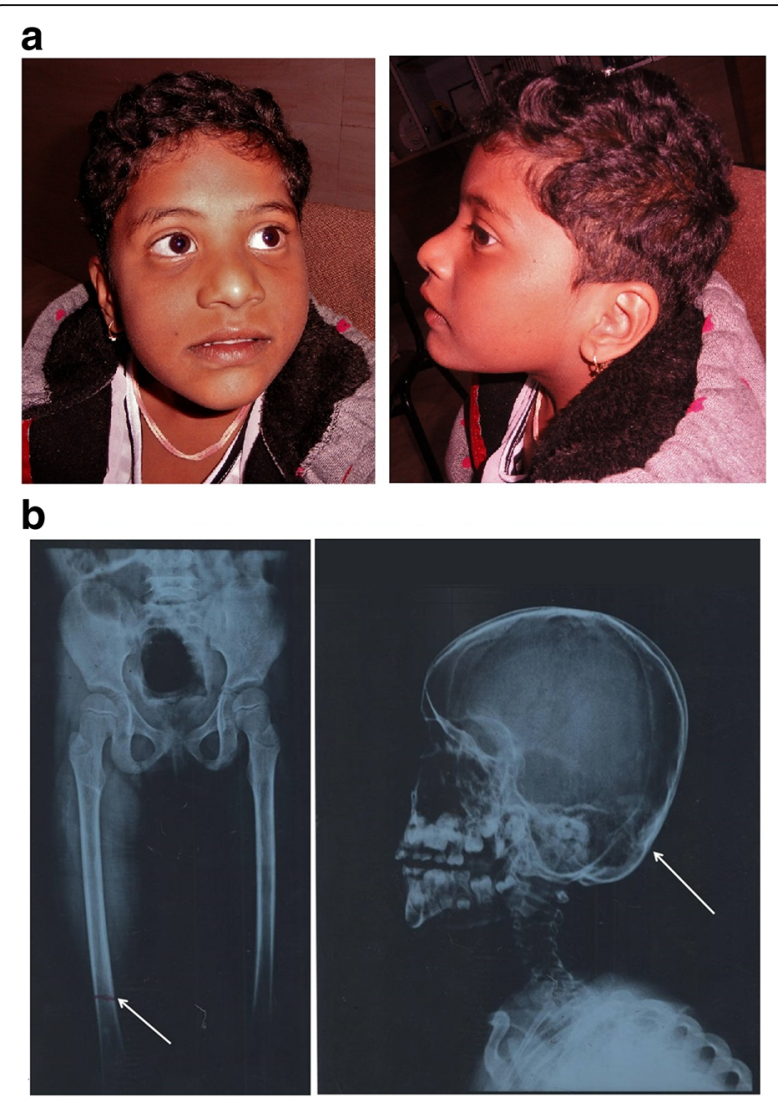

Fig. 1 a Clinical picture of the proband showing features of microcephaly, narrow bifrontal diameter, flat forehead, epicanthal folds, hypertelorism, depressed and low nasal bridge with bulbous nasal tip, flaring nares, prominent philtrum and pointed chin. b X-ray of extremities of the proband (Left): the arrow indicates small sclerotic areas observed in lower part of the right femur; X-ray of the skull (Right): abnormal skull shape skull; the arrow indicates minimal sclerosis of lower occipital region-bones periosteal reaction was noted. CT scan of the brain revealed prominent sulcal pattern with enlarged cisternal spaces in the brain parenchyma. These changes were prominently observed in the frontoparietal region. In addition, mild cortical atrophy was also detected. There was no evidence of focal hypo- or hyperdense lesion. Moreover, subdural, extradural or intracerebral hemorrhage, or subarachnoid bleeding was not observed. The pons, midbrain, both cerebellar hemisphere, and brain stem appeared normal. No mass lesion was observed in the posterior fossa.

MRI revealed a significant hypoplastic appearance of posterior part of the brain. It also showed corpus callosal dysgenesis with the absence of rostral area. Moreover, a mild paucity of the peritrigonal white matter was seen surrounding the trigones of bilateral lateral ventricles. However, no obvious gliosis, extra-axial collection or lesions were noted. Her echocardiogram showed normal systemic and pulmonary venous drainage, normal valves, and normal sized cardiac chambers. The Ophthalmological evaluation revealed bilateral intact vision with healthy macula.

\section{Molecular genetics investigations}

Chromosome banding study showed a normal female karyotype i.e. 46, XX at 550-bands resolution [20]. The fluorescence in situ hybridization (FISH) study was performed using LSI ELN (7q11) (orange) /LSI 7q22 (green) (Kreatech dual color probe). Since two signals were observed in all the cells, clinical prediction of Williams syndrome was ruled out. The proband DNA was further investigated with array comparative genomic hybridization $(\mathrm{aCGH})$ using [Agilent $60 \mathrm{~K}$ ], with an average resolution of $150 \mathrm{~kb}$. No copy number variations i.e. deletions and/or duplications of pathogenic significance were detected $[\operatorname{arr}(1-22, \mathrm{X}) \times 2]$. Thus, cytogenetic aberrations with resolution of $150 \mathrm{~kb}$ or greater were unlikely to be responsible for the clinical features in the proband.

The genomic DNA (gDNA) of the proband was isolated from the peripheral blood using the saltingout technique [31]. This DNA sample was processed for clinical exome sequencing (CES) on Illumina NextSeq 500. A detailed protocol is mentioned in the Additional file 1. Sequencing detected a homozygous missense variation c.1228 $\mathrm{T}>\mathrm{A}$ in the exon 6 of the FAM20C gene (OMIM*611061) (GenBank accession number NM.020223.3; coding sequences NP_064608). This variation resulted in the amino acid substitution of threonine for serine at codon 410 (p.Ser410Thr; ENST00000313766), confirming the clinical diagnosis of RS (OMIM \# 259775).

The functional effect of this variant was studied using the in silico analysis tools. The variant was predicted to be disease-causing by Mutation Taster (http://www. mutationtaster.org/), with a score of 58. The impact of the substitution of serine to threonine was predicted to be probably damaging (score of 0.972 ) by Polyphen2 (PolymorphismPhenotypingV2) (http://genetics.bwh.harvard. edu/pph2/). Scale-invariant feature transform (SIFT) (http://sift.jcvi.org/) predicted that the variant was tolerated with a score of 0.51, and PROVEAN anticipated that the variant had a deleterious effect on the protein function. This variant was reported as a likely pathogenic allele (SCV000583504.1) and benign (SCV000343602.2) in ClinVar. Its reference SNP number is rs148276213. The minor allele frequency of the rs148276213 variant is 0.0034 in the 1000 genomes database and 0.006108 in the ExAC database for the South Asian population.

CES also revealed another compound heterozygous variant in the tubulin gamma complex associated with protein 6 (TUBGCP6) gene (OMIM*610053) in the 
proband. The compound heterozygous variant in exon 24 [c.5327C > G (p.Ser1776Cys)] and exon 16 [c. $3383 \mathrm{G}>\mathrm{A}$ (p.Arg1128Lys)] is associated with microcephaly and chorioretinopathy-1 (OMIM\#251270). In silico analysis predicted the first variant (c.5327C > G/ p.Ser1776Cys) to be disease-causing using the Mutation Taster, tolerated by SIFT (score 0.07) and probably damaging by PolyPhen (score 1.00). While second variant (c.3383G > A /p.Arg1128Lys) found to be a polymorphism by Mutation T@ster, tolerated by SIFT (score 0.65), and benign by PolyPhen (score 0 . 023). Second variant was dismissed as the cause of disease in the proband, because this genotypic variation did not correlate with the phenotypic features of the proband, and also because various bioinformatics prediction softwares could not predict it as damaging.

The variant has an autosomal recessive mode of inheritance. Its confirmation in the proband and her parents was carried out using bi-directional Sanger sequencing with primers covering both the exon and the intron-exon boundary of exon 6 of the FAM20C gene (Additional file 2). Sequencing confirmed both the parents to be heterozygous, and the proband to be homozygous for c.1228 T > A (p.Ser410Thr) variant in exon 6 of FAM20C gene (Fig. 2a-d). This variant was submitted to ClinVar database (accession ID is SCV000583504.1). During a subsequent pregnancy, the

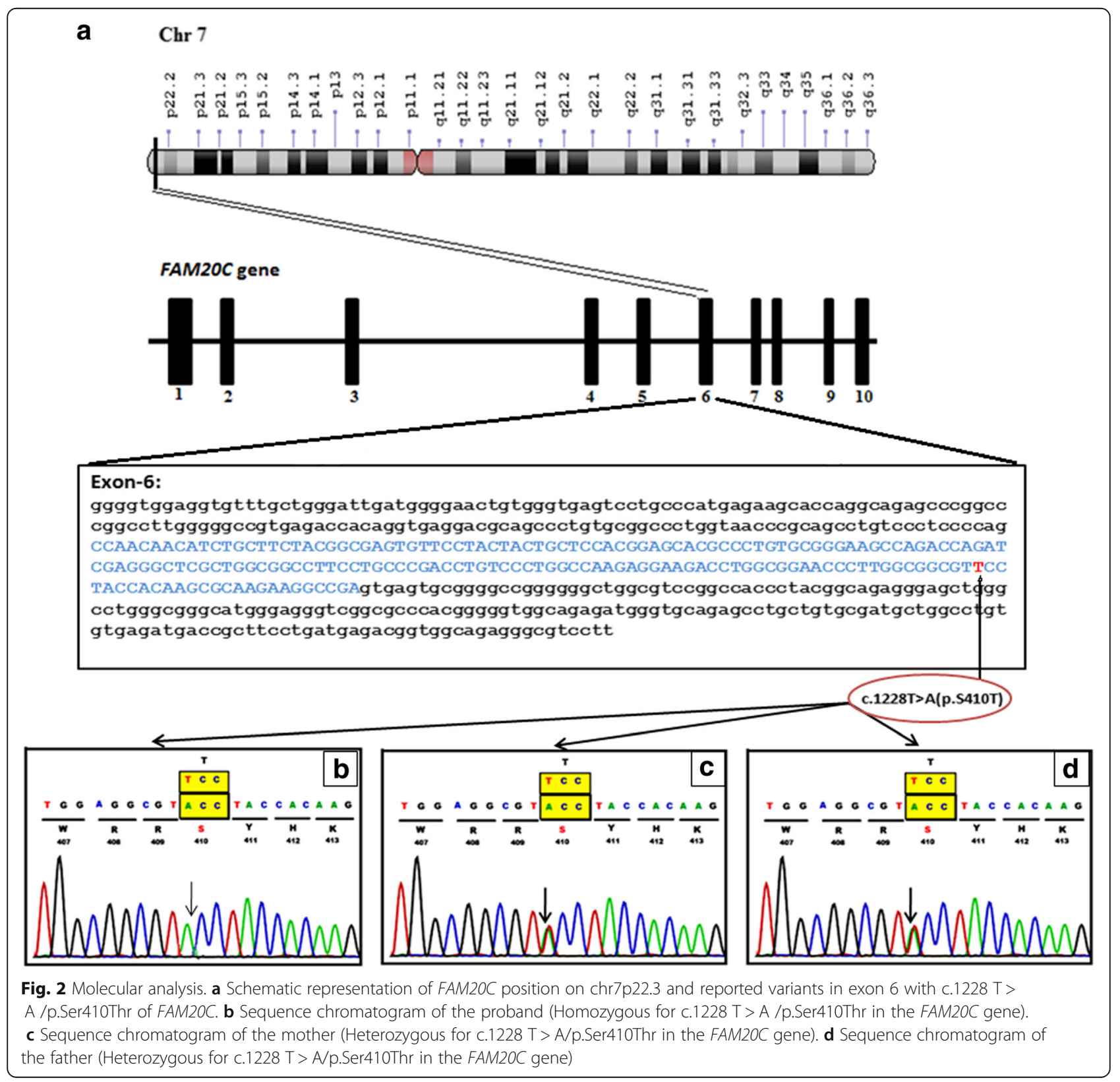


same family approached again for genetic counseling. The prenatal diagnosis revealed a heterozygous state of the fetus for the c.1228 T > A (p.Ser410Thr) variant, and a normal carrier child was delivered (data not shown).

Homology modeling, structure validation and protein stability due to c.1228 T > A (p.Ser410Thr) variant Using NCBI Basic Local Alignment Search Tool (BLAST), the native and mutated sequences of the FAM20C gene were studied to understand the effect of the variant, and also to predict the protein structure against PDB with default parameters [32]. The template PDBID: 5WRR was considered for modeling the protein structure. Additional details regarding the homological modeling is mentioned in the Additional file 3 [33-41].

A root mean sequence deviation (RMSD) of $0.3 \mathrm{~A}^{\circ}$ was observed between native and mutant structure, indicating changes in the loop regions of the superimposed structure. A decrease instability was predicted by iStable with a confidence score of 0.605 . Furthermore, I-Mutant predicted a large decrease in protein stability due to the variant p.Ser410Thr with $\Delta \Delta G$ value of $-1.37 \mathrm{Kcal} / \mathrm{mol}$ (Fig. 3).

\section{Conservation of the FAM20C p.Ser410Thr residue in orthologs}

The protein sequence of Homo sapiens (NP_064608) was aligned along with other species using an online multiple sequence alignment program known as Clastal Omega (https://www.ebi.ac.uk/Tools/msa/clustalo/). It is observed that the orthologs protein sequences of the FAM20C gene were highly identical to $H$. sapiens FAM20C gene protein sequence suggesting a highly conserved locus (Fig. 4a, b; Additional file 4).

\section{Population screening for c.1228T >A (p.Ser410Thr) variant by ARMS-PCR}

Using PCR technique of amplification-refractory mutation system (ARMS), 200 unrelated control samples (100 males and 100 females) were studied to demonstrate the pathogenicity of c.1228T $>$ A (p.Ser410Thr) variant. Amplification of the PCR product was confirmed by $2.5 \%$ agarose gel electrophoresis. From the 200 subjects screened for the above genotype, no carriers for the c.1228T $>$ A (p.Ser410Thr) variant were found (Additional file 5).

\section{Discussion and conclusions}

Most of the RS cases initially reported died at an infantile age. The prevalence of non-lethal RS has been reported in few cases over time. The most striking features of RS are osteosclerosis and facial anomalies [42]. The case presented here exhibited facial dysmorphism such as a flat forehead,

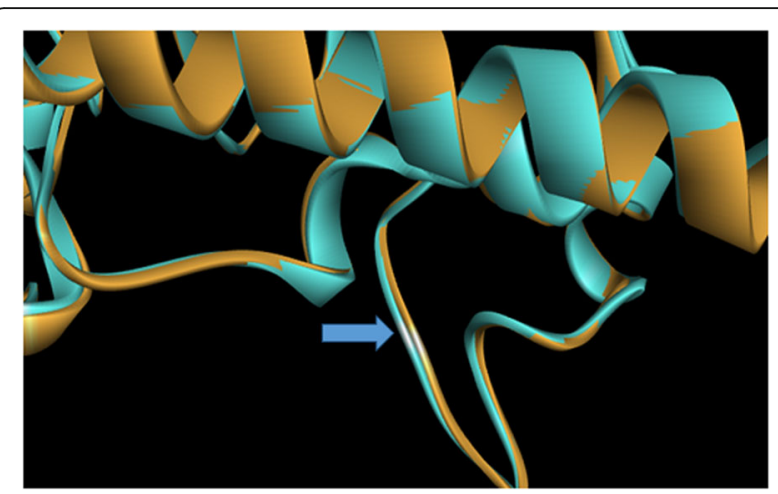

Fig. 3 Homology modeling, structure validation and protein stability due to c.1228 T > A (p.Ser410Thr) variant: The mutation has occurred in exon 6 of FAM2OC gene at codon number 410 causing codon change from TCC to ACC. The super imposed model of native structure (blue) and mutant structure (brown) produced using Discovery Studio software for mutation p.Ser410Thr shows conformational changes in the loop regions; indicated by blue arrow

epicanthal folds, hypertelorism, depressed and low nasal bridge with bulbous nasal tip, flaring nares, prominent philtrum, and pointed chin. Such phenotypical appearances have also been observed in other lethal and non-lethal RS cases $[7,8,20]$. These developmental delays was reported in two siblings affected with RS from two different families $[8,28]$. Developmental delay is a very rare phenomenon

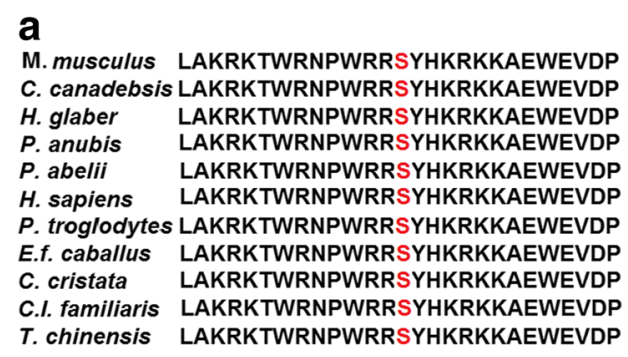

b NCBI Reference sequence

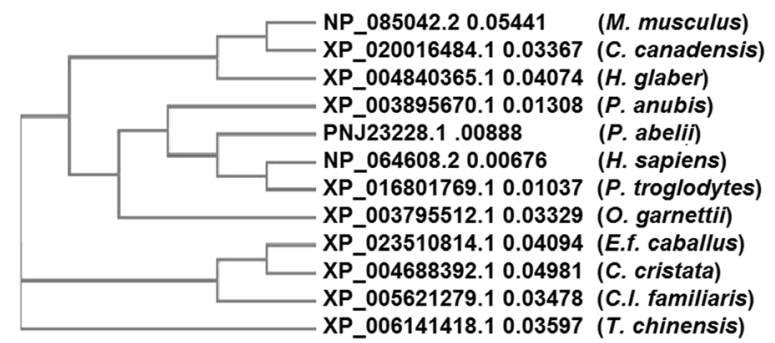

Fig. 4 Conservation of FAM2OC (p.Ser410Thr) residue in various orthologs: (a) The multiple alignment of the protein sequence region surrounding the variant c.1228 T > A (p.Ser410Thr) against various orthologous sequences. The conserved Serine (S) residue in all the orthologs is mark red. $\mathbf{b}$ The phylogenetic tree depicting the evolutionary conservation of FAM2OC gene in various orthologs 


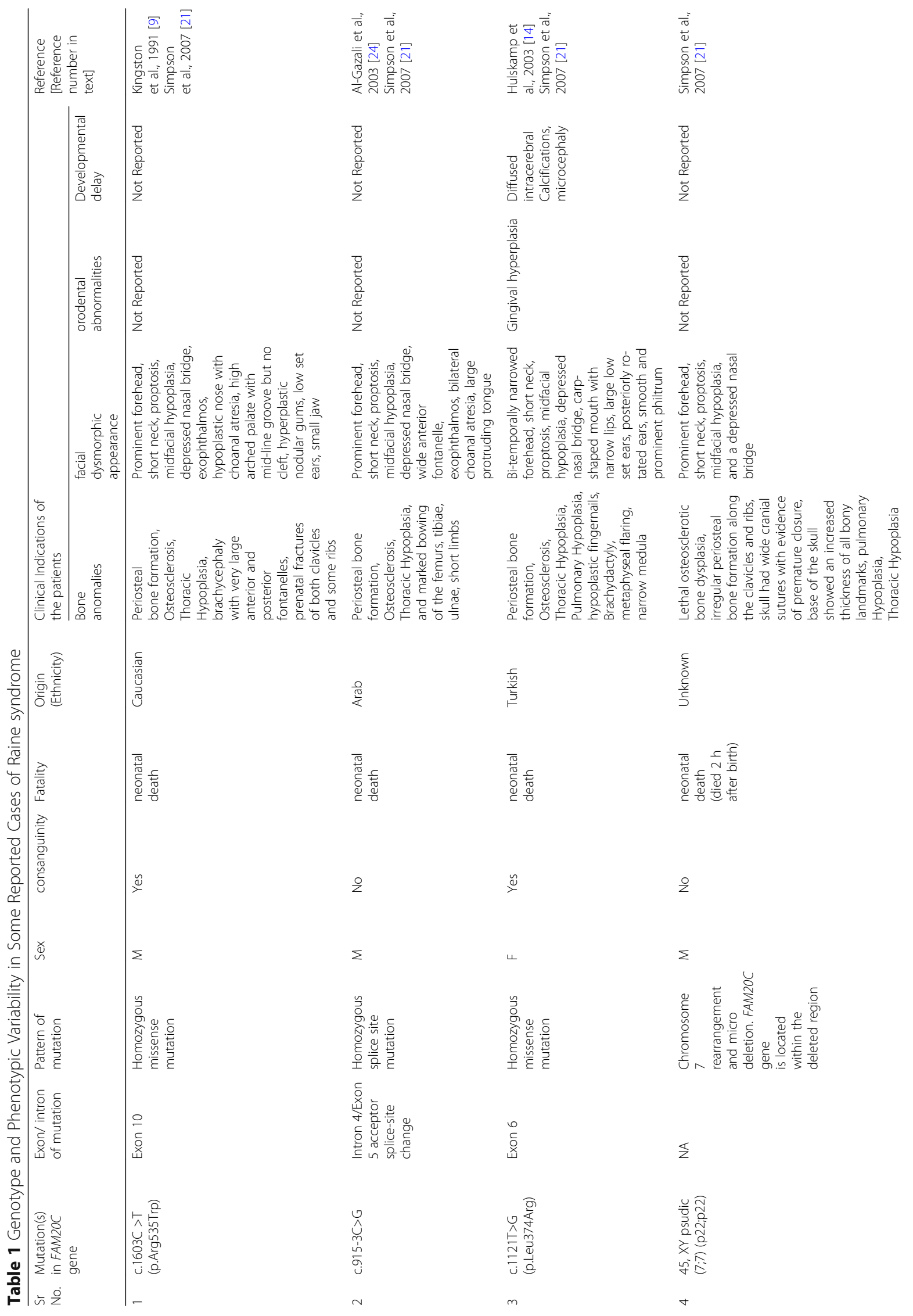




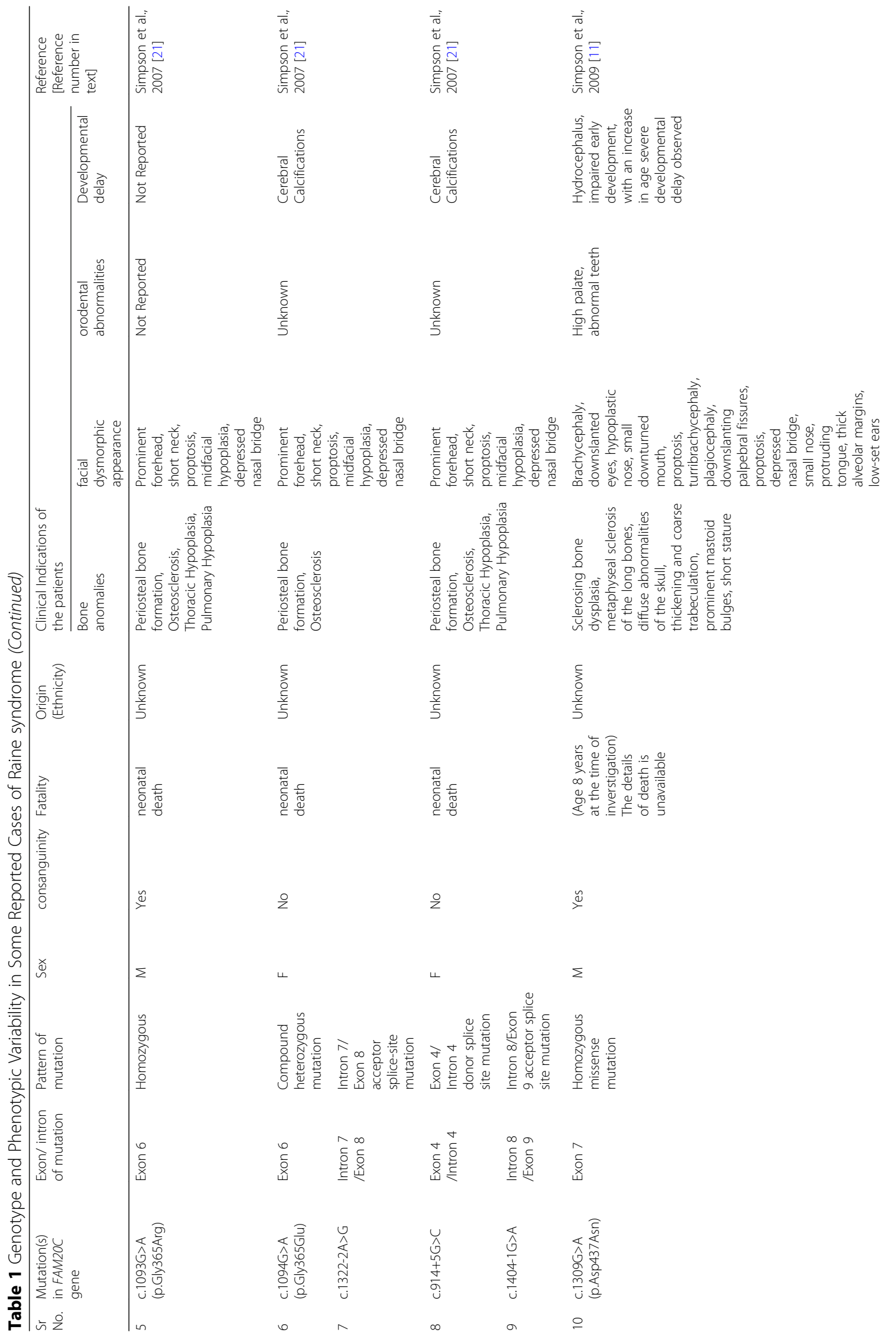




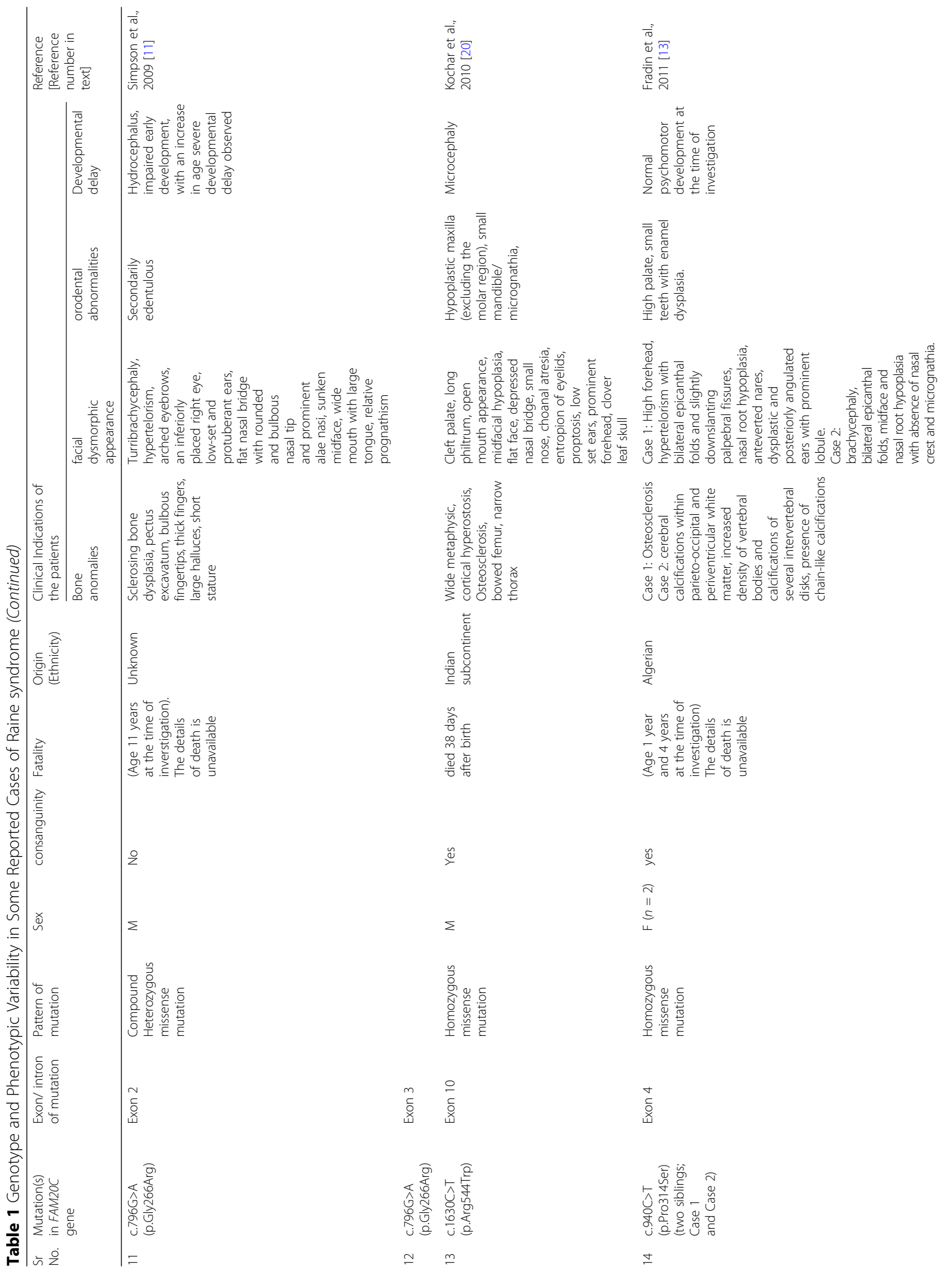




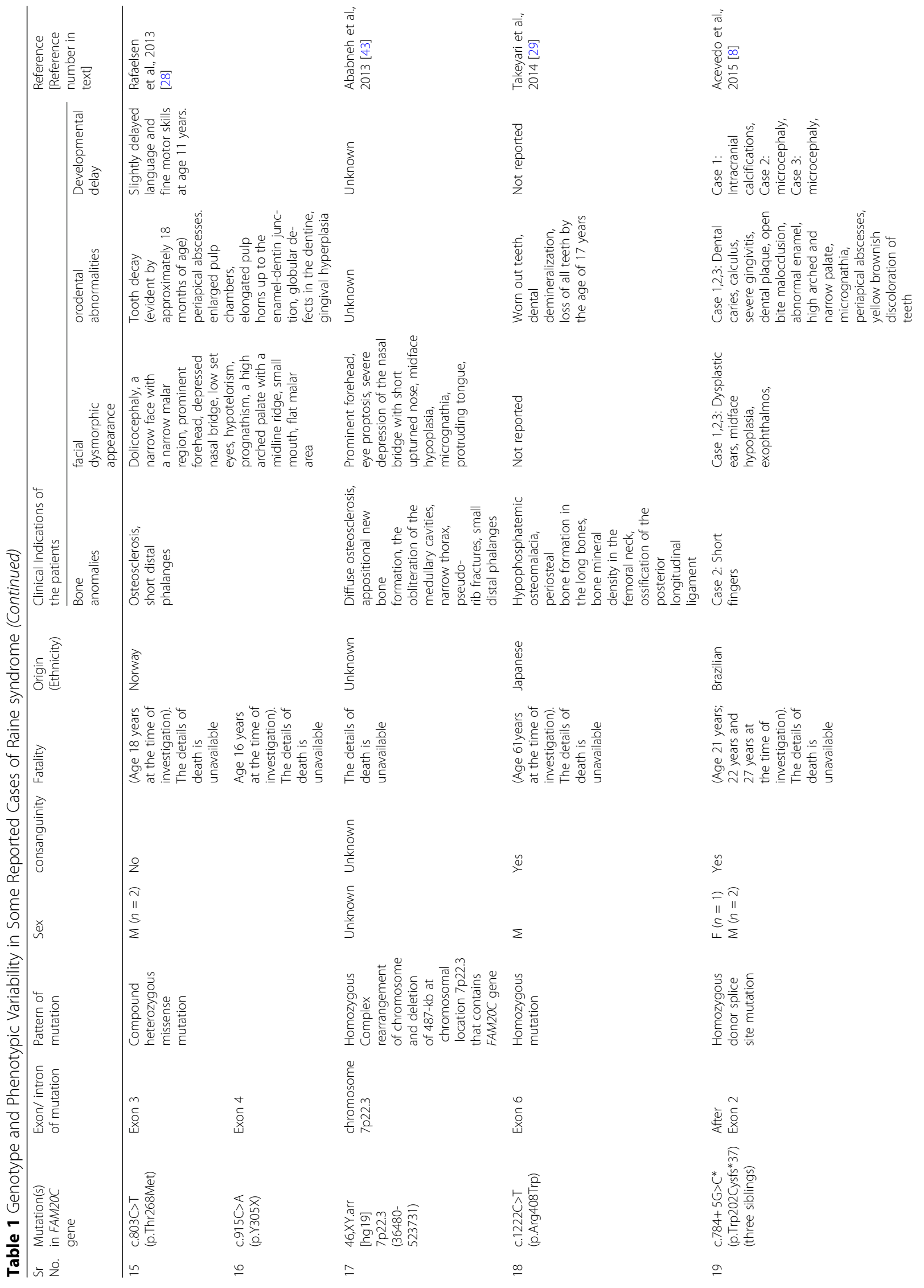




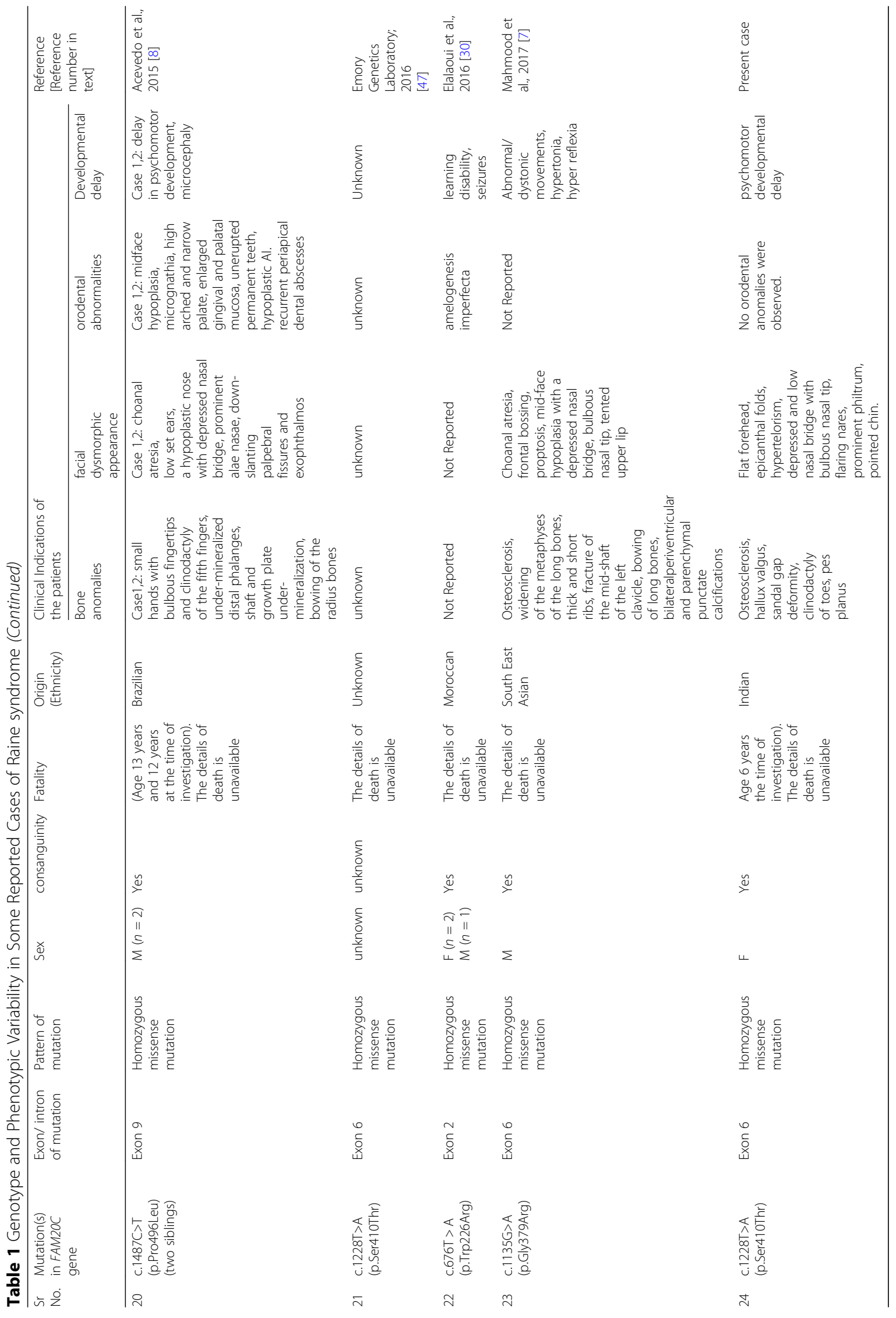


observed amongst RS patients. Additionally, bone abnormalities like osteosclerosis, hallux valgus, sandal gap deformity, clinodactyly of toes, and pes planus were observed. Several cases of RS reported so far, have shown a broad range of bone anomalies including thoracic hypoplasia, metaphyseal flaring and under-mineralized distal phalanges $[14,43]$. Thus, it can be concluded that there is an association of RS with heterogeneous clinical phenotypes.

Several mutations in the FAM20C gene have consistently been reported in RS patients (Table 1). In our case, homozygous missense variant in exon 6 of FAM20C gene was identified. The identified variant was also reported as benign by Emory University. Mutations in orthologs of the FAM20C gene have also exhibited a similar effect in different species. A group of Border Collies represented the canine model for human Raine syndrome. A recessive transmission of c.899C $>$ T (p.A300V) mutation in FAM20C gene resulted in mineralization defect in these canines [44]. In a murine model, specific ablation of the FAM20C gene in cells expressing type I collagen led to skeletal defects and hypophosphatemia [45]. This suggests that FAM20C gene function is highly conserved amongst orthologs.

Another compound heterozygous variation in the TUBGCP6 gene (OMIM*610053) was observed in the current proband. Mutation in this gene caused microcephaly and chorioretinopathy-1 (OMIM\#251270). The prominent clinical feature for this disease was microcephaly, retinal pigmentary abnormalities, and early onset of visual impairment. The proband in the present case had microcephaly with intact vision, ruling out retinopathy. There was a lack of genotypic and phenotypic co-relation in the present variant, and the variant was not considered to be predisposing for the clinical features of the proband.

One of the most prominent clinical indications in RS are skeletal defects. Mutation/s in FAM20C lead to loss of function, resulting in hypophosphatemia. The tissuenonspecific isoenzyme of alkaline phosphatase (TNSALP) substrates together with inorganic pyrophosphate gets accumulated in the extracellular niche due to mutation/s in FAM20C gene. This causes hindrance in the normal function of mineralization. An excess of pyrophosphate accumulation results in tooth loss, osteomalacia, and calcification of bones. Asfotase alfa, a hydroxyapatite-targeted recombinant TNSALP is being used as an enzyme replacement therapy to get rid of excess pyrophosphate [46]. However, the enzyme replacement therapy is expensive and may not be affordable to many families. In such a scenario, precise genetic counseling and prenatal diagnosis remains the preferred choice.

Though RS is synonymously read as lethal osteosclerotic bone dysplasia, several reported cases including ours show the non-lethal clinical phenotype associated with the FAM20C mutation. In these mild RS cases, the patient survives beyond infancy. RS reflects marked variability in presentations ranging from severe to localized, non-diffuse, and mild bone osteosclerosis. Facial appearance and striking radiological findings prompt the clinical diagnosis of RS. More number of such cases is crucial to define the variable radiologic, metabolic, and molecular defects of this seemingly lethal and non-lethal autosomal recessive syndrome.

\section{Additional files}

Additional file 1: Molecular investigations. The file describes the method used in isolation of genomic DNA, NGS and bioinformatics tools used during analysis. (DOCX $16 \mathrm{~kb}$ )

Additional file 2: Sanger sequencing (Variant Confirmation Test). It describes the details of primer's used during Sanger sequencing of the proband and parents (DOCX $15 \mathrm{~kb}$ )

Additional file 3: Homology modeling, structure validation and protein stability due to c.1228T>A (p.Ser410Thr) variant. File describes the influence of variant change on the protein structure (DOCX $16 \mathrm{~kb}$ )

Additional file 4: Conservation of the FAM20C p.Ser410Thr residue in orthologs. Conservation of the variant in orthologs and Homo sapiens. (DOCX $15 \mathrm{~kb}$ )

Additional file 5: Population screening for c.1228T>A (p.Ser410Thr) variant by ARMS-PCR. The file provides details of normal healthy individuals studied for variant (c.1228T>A (p.Ser410Thr)) by ARMS-PCR. (DOCX $15 \mathrm{~kb}$ )

\section{Abbreviations}

1,25(OH) 2 D: 1,25-Dihydroxy vitamin D; aCGH: Array Comparative Genomic Hybridization; ADHD: Attention Deficit Hyperactivity Disorder;

ARMS: Amplification-refractory mutation system; CES: Clinical Exome Sequencing; CNS: Central Nervous System; Dmp1: Dentin matrix protein 1; EEG: Electroencephalogram; ELN: Elastin; ExAC: The Exome Aggregation Consortium; FAM20C: Family with sequence similarity 20,member C; FGF23: Fibroblast growth factor 23; FISH: Fluorescence in Situ Hybridization; IUGR: Intrauterine Growth Retardation; LVEF: Left ventricular ejection fraction; NCBI: National Centre for Biotechnology Information; OMIM: Online Mendelian Inheritance in Man; PCR: Polymerase chain reaction; PDB: Protein Data Base; RMSD: root mean sequence deviation; SAVES: Structural Analysis and Verification Server; SCPP: Secretory calcium binding-phosphoproteins; SIBLINGs: Small integrin-binding ligand, N-linked glycoproteins; SIFT: Scaleinvariant feature transform; TNSALP: Tissue-nonspecific isoenzyme of alkaline phosphatase; TUBGCP6: Tubulin gamma complex associated protein 6; YASARA: Yet Another Scientific Artificial Reality Application

\section{Acknowledgments}

We thank Dr. Lubna Naznin for her partial help in preparing the manuscript and Dr. K. Bhanuprakash Achary for doing protein Homology modeling. We thank Dr. Harsh Sheth, Riddhi Sheth and Dr. Sunil Trivedi for critical reading, Dr. Krati Shah and Dr. Mehul Mistri for evaluating the manuscript. We show our gratitude to the proband and the family for their support - without their consent, this study would not have been possible. We acknowledge the Gujarat State Biotech Mission (GSBTM), Govt. of Gujarat for partial support to the work [grant no.: GSBTM/MD/PROJECTS/SSA/505/4865/2016-17].

\section{Funding}

This work is partially supported by Gujarat State Biotechnology Mission (GSBTM) [grant no:: GSBTM/MD/PROJECTS/SSA/505/4865/2016-17]. The funding agency was not directly or indirectly involved in the study design, specimen collection, analysis, interpretation and preparation of the manuscript. 


\section{Availability of data and materials}

The dataset generated and/or analyzed during the current study is available in the ClinVar repository, https://www.ncbi.nlm.nih.gov/clinvar/variation/ 289269/.

ClinVar Accession ID for c.1228T>A (p.Ser410Thr) variant in Exon 6 of FAM20C gene: SCV000583504.1 [47].

\section{Authors' contributions}

Conceived and designed the experiments: JS. Clinical analysis: JS and AG. Wrote the revised draft of the manuscript: DP, Laboratory workup: DP and RB. Made critical revisions and approved final version: JS and FS. All authors reviewed and approved the final manuscript.

\section{Ethics approval and consent to participate}

The present case report has been approved by the institutional ethics committee [FRIGE's Institute of Human Genetics] with approval number FRIGE/IEC/14/2016 dated 19th November 2016. This process is in accordance with the declaration of Helsinki.

- An informed consent for investigation and publication was obtained from the proband father at the time of enrollment for the study [This was in accordance with the requirement of the institutional ethics committee].

\section{Consent for publication}

Informed written consent was obtained from parents on behalf of the minor child for publication of their clinical details and/or clinical images. A copy of the written consent is available for review.

\section{Competing interests}

The authors declare that they have no competing interests (financial or non-financial) in the present study.

\section{Publisher's Note}

Springer Nature remains neutral with regard to jurisdictional claims in published maps and institutional affiliations.

\section{Author details}

${ }^{1}$ FRIGE's Institute of Human Genetics, FRIGE House, Jodhpur Gam Road, Satellite, Ahmedabad 380015, India. ${ }^{2}$ Unique Hospital, Main Road, South Kasba, Solapur 413007, India.

\section{Received: 7 September 2017 Accepted: 25 April 2018}

\section{Published online: 11 May 2018}

\section{References}

1. Faundes V, Castillo-Taucher S, Gonzalez-Hormazabal P, Chandler K, Crosby A Chioza B. Raine syndrome: an overview. Eur J Med Genet. 2014;57:536-42.

2. Tagliabracci VS, Engel JL, Wen J, Wiley SE, Worby CA, Kinch LN, et al. Secreted kinase phosphorylates extracellular proteins that regulate biomineralization. Science. 2012;336:1150-3.

3. Oya K, Ishida K, Nishida T, Sato S, Kishino M, Hirose K, et al. Immunohistochemical analysis of dentin matrix protein 1 (Dmp1) phosphorylation by Fam20C in bone: implications for the induction of biomineralization. Histochem Cell Biol. 2017;147:341-51.

4. Kinoshita Y, Hori M, Taguchi M, Fukumoto S. Functional analysis of mutant FAM20C in Raine syndrome with FGF23-related hypophosphatemia. Bone. 2014;67:145-51.

5. Raine J, Winter RM, Davey A, Tucker SM. Unknown syndrome: microcephaly, hypoplastic nose, exophthalmos, gum hyperplasia, cleft palate, low set ears, and osteosclerosis. J Med Genet. 1989;26:786-8.

6. Kan AE, Kozlowski K. New distinct lethal osteosclerotic bone dysplasia (Raine syndrome). Am J Med Genet. 1992;43:860-4.

7. Mahmood N, Donne A, Weber A, Dharmaraj P. Raine syndrome: a review and a report of metabolic bone disease as a new link. Research. 2014; https://doi.org/10.13070/rs.en.1.890.

8. Acevedo AC, Poulter JA, Alves PG, de Lima CL, Castro LC, Yamaguti PM, et al. Variability of systemic and oro-dental phenotype in two families with non-lethal Raine syndrome with FAM20C mutations. BMC Med Genet. 2015;16:8.
9. Kingston HM, Freeman JS, Hall CM. A new lethal sclerosing bone dysplasia. Skelet Radiol. 1991;20:117-9.

10. Al Mane K, Coates R, McDonald P. Intracranial calcification in Raine syndrome. Pediatr Radiol. 1996;26:55-8.

11. Simpson M, Scheuerle A, Hurst J, Patton M, Stewart H, Crosby A. Mutations in FAM20C also identified in non-lethal osteosclerotic bone dysplasia. Clin Genet. 2009;75:271-6.

12. Koob M, Doray B, Fradin M, Astruc D, Dietemann JL. Raine syndrome: expanding the radiological spectrum. Pediatr Radiol. 2011;41:389-93.

13. Fradin M, Stoetzel C, Muller J, Koob M, Christmann D, Debry C, et al. Osteosclerotic bone dysplasia in siblings with a Fam20C mutation. Clin Genet. 2011;80:177-83.

14. Hulskamp G, Wieczorek D, Rieder H, Louwen F, Hörnig-Franz I, Rickert CH, et al. Raine syndrome: report of a family with three affected sibs and further delineation of the syndrome. Clin Dysmorphol. 2003;12:153-60.

15. Rickert CH, Rieder H, Rehder H, Hulskamp G, Hörnig-Franz I, Louwen F, et al. Neuropathology of Raine syndrome. Acta Neuropathol. 2002;103:281-7.

16. Acosta AX, Peres LC, Chimelli LC, Pina-Neto JM. Raine dysplasia: a Brazilian case with a mild radiological involvement. Clin Dysmorphol. 2000;9:99-101.

17. Al-Mane K, Al-Dayel F, McDonald P. Intracranial calcification in Raine syndrome: radiological pathological correlation. Pediatr Radiol. 1998;28:820-3.

18. Shalev SA, Shalev E, Reich D, Borochowitz ZU. Osteosclerosis, hypoplastic nose, and proptosis (Raine syndrome): further delineation. Am J Med Genet. 1999;86:274-7.

19. Mahafza T, El-Shanti H, Omari H. Raine syndrome: report of a case with hand and foot anomalies. Clin Dysmorphol. 2001;10:227-9.

20. Kochar GS, Choudhary A, Gadodia A, Gupta N, Simpson MA, Crosby AH, et al. Raine syndrome: a clinical, radiographic and genetic investigation of a case from the Indian subcontinent. Clin Dysmorphol. 2010;19:153-6.

21. Simpson MA, Hsu R, Keir LS, Hao J, Sivapalan G, Ernst LM, et al. Mutations in FAM20C are associated with lethal osteosclerotic bone dysplasia (Raine syndrome), highlighting a crucial molecule in bone development. Am J Hum Genet. 2007;81:906-12.

22. Rejjal A. Raine syndrome. Am J Med Genet. 1998;78:382-5.

23. Kondoh T, Matsumoto T. Raine syndrome. Ryoikibetsu Shokogun Shirizu. 2001;34:569-70.

24. Al-Gazali LI, Jehier K, Nazih B, Abtin F, Haas D, Sadagahatian R. Further delineation of Raine syndrome. Clin Dysmorphol. 2003;12:89-93.

25. Gunes T, Kurtoglu S, Cetin N, Oztürk MA, Topaloglu N. Raine syndrome associated with cytomegalovirus infection. Turk J Pediatr. 2005;47:89-91.

26. Chitayat D, Shannon P, Keating S, Toi A, Blaser S, Friedberg T, et al. Raine syndrome: a rare lethal osteosclerotic bone dysplasia. Prenatal diagnosis, autopsy, and neuropathological findings. Am J Med Genet A. 2007;143: 3280-5.

27. Ababneh FK, Alswaid A, Youssef T, Al Azzawi M, Crosby A, Albalwi MA. Hereditary deletion of the entire FAM20C gene in a patient with Raine syndrome. Am J Med Genet A. 2013;161A:3155-60.

28. Rafaelsen SH, Raeder H, Fagerheim AK, Knappskog P, Carpenter TO, Johansson S, et al. Exome sequencing reveals FAM20c mutations associated with fibroblast growth factor 23-related hypophosphatemia, dental anomalies, and ectopic calcification. J Bone Miner Res. 2013;28:1378-85.

29. Takeyari S, Yamamoto T, Kinoshita Y, Fukumoto S, Glorieux FH, Michigami T, et al. Hypophosphatemic osteomalacia and bone sclerosis caused by a novel homozygous mutation of the FAM20C gene in an elderly man with a mild variant of Raine syndrome. Bone. 2014;67:56-62.

30. Elalaoui SC, Al-Sheqaih N, Ratbi I, Urquhart JE, O'Sullivan J, Bhaskar S, et al. Non lethal Raine syndrome and differential diagnosis. Eur J Med Genet. 2016:59:577-83.

31. Miller SA, Dykes DD, Polesky HF. A simple salting out procedure for extracting DNA from human nucleated cells. Nucleic Acids Res. 1988;16:1215.

32. Altschul SF, Gish W, Miller W, Myers EW, Lipman DJ. Basic local alignment search tool. J Mol Biol. 1990;215:403-10.

33. Arnold K, Bordoli L, Kopp J, Schwede T. The SWISS-MODEL workspace: a web-based environment for protein structure homology modeling. Bioinformatics. 2006;22:195-201.

34. Schwede T, Kopp J, Guex N, Peitsch MC. SWISS-MODEL: an automated protein homology-modeling server. Nucleic Acids Res. 2003;31:3381-5.

35. Krieger E, Joo K, Lee J, Lee J, Raman S, Thompson J, et al. Improving physical realism, stereochemistry, and side-chain accuracy in homology 
modeling: four approaches that performed well in CASP8. Proteins. 2009; 77(Suppl 9):114-22.

36. Bowie JU, Luthy R, Eisenberg D. A method to identify protein sequences that fold into a known three-dimensional structure. Science. 1991;253:164-70.

37. Luthy R, Bowie JU, Eisenberg D. Assessment of protein models with threedimensional profiles. Nature. 1992;356:83-5.

38. Colovos C, Yeates TO. Verification of protein structures: patterns of nonbonded atomic interactions. Protein Sci. 1993;2:1511-9.

39. Laskowski RA, MacArthur MW, Moss DS, Thornton JM. PROCHECK - a program to check the stereochemical quality of protein structures. J Appl Crystallogr. 1993;26:283-91.

40. Chi-Wei C, Jerome L, Yen-Wei C. iStable: off-the-shelf predictor integration for predicting protein stability changes. BMC Bioinformatics. 2013;14 (suppl 2):S5.

41. Capriotti E, Fariselli P, Casadio R. I-Mutant2.0: predicting stability changes upon mutation from the protein sequence or structure. Nucleic Acids Res. 2005;33:306-10.

42. Whyte MP, McAlister WH, Fallon MD, Pierpont ME, Bijanki VN, Duan S, et al. Raine syndrome (OMIM \#259775), caused by FAM20C mutation, is congenital Sclerosing Osteomalacia with cerebral calcification (OMIM 259660). J Bone Miner Res. 2017:32:757-69.

43. Ababneh FK, AlSwaid A, Youssef T, Al Azzawi M, Crosby A, AlBalwi MA. Hereditary deletion of the entire FAM2OC gene in a patient with Raine syndrome. Am J Med Genet A. 2013;161:3155-60.

44. Hytönen MK, Arumilli M Lappalainen AK, Owczarek-Lipska M, Jagannathan $\checkmark$, Hundi S, et al. Molecular characterization of three canine models of human rare bone diseases: Caffey, van den Ende-Gupta, and Raine syndromes. PLoS Genet. 2016; https://doi.org/10.1371/journal.pgen.1006037.

45. Liu P, Ma S, Zhang H, Liu C, Lu Y, Chen L, et al. Specific ablation of mouse Fam20C in cells expressing type I collagen leads to skeletal defects and hypophosphatemia. Sci Rep. 2017; https://doi.org/10.1038/s41598-017-03960-x.

46. Whyte MP. Hypophosphatasia: enzyme replacement therapy brings new opportunities and new challenges. J Bone Miner Res. 2017;32:667-75.

47. https://www.ncbi.nlm.nih.gov/clinvar/16376712/ accessed on 07 Sept 2017.

\section{Ready to submit your research? Choose BMC and benefit from:}

- fast, convenient online submission

- thorough peer review by experienced researchers in your field

- rapid publication on acceptance

- support for research data, including large and complex data types

- gold Open Access which fosters wider collaboration and increased citations

- maximum visibility for your research: over $100 \mathrm{M}$ website views per year

At BMC, research is always in progress.

Learn more biomedcentral.com/submissions 